\title{
Influence of common synaptic input to motor neurons on the neural drive to
} muscle in essential tremor

\author{
Juan A. Gallego, Jakob L. Dideriksen, Ales Holobar, Jaime Ibáñez, José L. Pons, Elan D. Louis, \\ Eduardo Rocon, \\ and Dario Farina
}

Gallego JA, Dideriksen JL, Holobar A, Ibáñez J, Pons JL, Louis ED, Rocon E, Farina D. Influence of common synaptic input to motor neurons on the neural drive to muscle in essential tremor. $J$ Neurophysiol 113: 182-191, 2015. First published October 1, 2014; doi: $10.1152 / \mathrm{jn} .00531 .2014$.- Tremor in essential tremor (ET) is generated by pathological oscillations at $4-12 \mathrm{~Hz}$, likely originating at cerebello-thalamo-cortical pathways. However, the way in which tremor is represented in the output of the spinal cord circuitries is largely unknown because of the difficulties in identifying the behavior of individual motor units from tremulous muscles. By using novel methods for the decomposition of multichannel surface EMG, we provide a systematic analysis of the discharge properties of motor units in nine ET patients, with concurrent recordings of EEG activity. This analysis allowed us to infer the contribution of common synaptic inputs to motor neurons in ET. Motor unit short-term synchronization was significantly greater in ET patients than in healthy subjects. Furthermore, the strong association between the degree of synchronization and the peak in coherence between motor unit spike trains at the tremor frequency indicated that the high synchronization levels were generated mainly by common synaptic inputs specifically at the tremor frequency. The coherence between EEG and motor unit spike trains demonstrated the presence of common cortical input to the motor neurons at the tremor frequency. Nonetheless, the strength of this input was uncorrelated to the net common synaptic input at the tremor frequency, suggesting a contribution of spinal afferents or secondary supraspinal pathways in projecting common input at the tremor frequency. These results provide the first systematic analysis of the neural drive to the muscle in ET and elucidate some of its characteristics that determine pathological tremulous muscle activity.

pathological tremor; motor unit; motor neuron; coherence; EMG; EEG

ESSENTIAL TREMOR (ET) is characterized by $4-$ to $12-\mathrm{Hz}$ upper limb tremor during posture and movement (Benito-León and Louis 2006). Tremor in ET is ultimately generated by the abnormal rhythmic entrainment of motor neurons innervating the affected muscles (Elble and Deuschl 2009), which results from the combination of central oscillatory activity (at cer- ebello-thalamo-cortical pathways and possibly other structures; Benito-León and Louis 2006), reflex loops with different arc length, and limb properties (Deuschl et al. 2001; McAuley and Marsden 2000). The manner in which these mechanisms interact to generate the abnormal neural activity is not fully understood (Louis et al. 2013), partly because of the difficulty in directly recording the output of spinal motor neurons activating the tremulous muscles (neural drive to muscles).

Motor unit spike trains have been traditionally analyzed with intramuscular electrodes, a technique that suffers from several limitations, especially when applied in pathological conditions such as tremor. One of these limitations is the small number of identified motor units, which often does not comprise a representative sample of the active population (Merletti and Farina 2009). Moreover, the invasiveness of the technique and the sensitivity to small electrode movements strongly limit its applicability in the investigation of tremor. Indeed, only one study, to our knowledge, has reported motor neuron discharge properties in ET using this technique (Elek et al. 1991), focusing on the tendency of individual motor neurons to fire paired or tripled discharges with short interspike epochs (ISIs; $\sim 10-90 \mathrm{~ms}$ ). These paired discharges likely occur because of the presence of a large excitatory drive (Kudina and Andreeva 2013) and are thus etiologically different from double discharges or doublets, which arise during delayed depolarization (Kudina and Andreeva 2013) and have briefer ISIs ( $<10 \mathrm{~ms}$; Heckman and Enoka 2012).

Despite the lack of direct measurements of motor unit behavior in tremor, there are long-standing assumptions on some of the motor unit properties in ET. For example, it is generally assumed that motor units in ET patients are highly synchronized (Elble and Deuschl 2009), although this assumption has never been experimentally verified. If confirmed, the presence of high synchronization among motor units would imply high levels of common synaptic inputs to motor neurons, which may have cortical (Farmer et al. 1993), subcortical (Boonstra et al. 2008), or afferent (Dartnall et al. 2008) origin.

In this study, we provide a systematic analysis of the discharge properties of motor units in ET patients, with concurrent recordings of EEG activity. Spike trains of individual motor units were identified with a novel algorithm for decom- 
posing multichannel surface EMG (Holobar et al. 2012), which permitted us to reliably detect several motor units accurately and noninvasively. The availability of this technique provides the unique possibility to precisely assess for the first time the neural drive to muscle in ET. With this approach we aimed to first directly measure the levels of motor neuron synchronization in ET and to further investigate the strength and source of common synaptic inputs to the motor neuron pool with coherence analysis, both between motor unit spike trains and between EEG and motor unit spike trains. These analyses provide a systematic insight into the properties of the neural drive to the muscle in ET and elucidate the causes of specific components of common input determining pathological tremulous muscle activity.

\section{MATERIALS AND METHODS}

Subjects. We present results for nine patients [5 women, 4 men; age $($ mean $\pm \mathrm{SD}): 71.0 \pm 5.6 \mathrm{yr}$, range $64-80 \mathrm{yr}]$ with a diagnosis of definite ET according to the criteria of the Tremor Investigation Group and the consensus of the Movement Disorder Society Group (Deuschl et al. 1998). All patients showed visible and persistent postural and kinetic tremor of the arms (unilateral or bilateral) and, in some cases, also at rest. No patient exhibited head or trunk tremor during the examination or had a history of neurological diseases other than ET. None had features of parkinsonism (bradykinesia, rigidity) aside from isolated rest tremor. Mean disease duration was $22.7 \pm$ $10.0 \mathrm{yr}$ (range $8-36 \mathrm{yr}$ ). Tremor severity ranged from mild to severe, with a mean score in the most affected limb of $24.7 \pm 7.0$ (range 14-32), according to the Fahn-Tolosa-Marin scale. Four patients were taking antitremor drugs (propranolol $120 \mathrm{mg}, 1$ patient; propranolol sporadically, 1 patient; propranolol $60 \mathrm{mg}$ and clonazepam $0.5 \mathrm{mg}, 1$ patient; propranolol $80 \mathrm{mg}, 1$ patient; all values indicate daily dosage), which in all cases were withheld for at least $12 \mathrm{~h}$ before the recordings. Patients were selected for enrollment by neurologists at two locations ( 3 at Hospital General Universitario, Valencia, Spain, and 6 at Hospital Universitario "12 de Octubre," Madrid, Spain), starting 3 mo before the experiments. They were identified from the databases of patients from both hospitals after in-patient examination. No patient declined to participate in the study.

Ethical approval. The local ethical committees at Hospital Universitario "12 de Octubre," Madrid, Spain, and Universidad Politécnica de Valencia, Valencia, Spain, gave approval to the study and warranted its accordance with the Declaration of Helsinki. All patients were informed beforehand and gave written informed consent to participate.

Recordings. Hand tremor at the most affected side (defined in situ after examination by a trained practitioner) was concurrently recorded with surface EMG and solid-state gyroscopes. Surface EMG was recorded with a $13 \times 5$ electrode grid with an interelectrode distance of $8 \mathrm{~mm}$ (LISiN-OT Bioelettronica, Turin, Italy). The grid was placed over the extensors of the wrist, centered laterally above the extensor digitorum communis, and longitudinally above the muscle belly; a wrist bracelet soaked in water served as common reference. The signal was amplified (EMGUSB, OT Bioelettronica), band-pass filtered $(10-750 \mathrm{~Hz})$, and sampled at $2,048 \mathrm{~Hz}$ by a 12 -bit A/D converter. Hand movement was measured with a pair of solid-state gyroscopes (Technaid, Madrid, Spain) placed on the dorsum of the hand and the distal third of the forearm, by computing their difference (Gallego et al. 2010; Rocon et al. 2006). The raw gyroscope signals were sampled at $50 \mathrm{~Hz}$ by a 12 -bit A/D converter and low-pass filtered $(<20 \mathrm{~Hz})$. At the same time, EEG signals were recorded from 32 positions of the scalp, following the International 10-20 System (AFz, F3, F1, Fz, F2, F4, FC5, FC3, FC1, FCz, FC2, FC4, FC6, C5, C3, C1, Cz, C2, C4, C6, CP5, CP3, CP1, CPz, CP2, CP4, CP6, P3, P1, Pz, P2, and P4), with passive Au electrodes. The reference was set to the common potential of the two earlobes, and $\mathrm{Az}$ was used as ground. The signal was amplified (gUSBamp, g.Tec, Graz, Austria), band-pass $(0.1-60 \mathrm{~Hz})$ and notch $(50 \mathrm{~Hz})$ filtered, and sampled at $256 \mathrm{~Hz}$ by a 16 -bit A/D converter. The recording systems were synchronized with a common clock signal generated by the computer acquiring the gyroscope data. The experiments were performed at Instituto de Biomecánica de Valencia, Valencia, Spain (patients 01-03) and Hospital Universitario "12 de Octubre," Madrid, Spain (patients 04-09). The data were stored and analyzed off-line with MATLAB (The MathWorks, Natick, MA). Figure 1, $A-C$, show representative EEG, surface EMG, and gyroscope signals.

Procedure. The recordings were performed while patients were seated in a comfortable armchair in a dimly illuminated room. Postural or rest tremor (depending on the patient) was elicited by asking the patients to keep the hands outstretched with palms down, parallel to the ground, while the forearms were fully supported on an armrest, or by asking them to relax with the hands hanging freely. The patients were instructed to stay relaxed and keep their gaze fixed on a wall at $\sim 2$-m distance, and those with mild tremor severity were asked to mentally count backward during the recordings to enhance their tremor (Hellwig et al. 2001).

Patients performed a series of 4-min trials (between 1 and 3, depending on how they tolerated the setup and on the quality of the recordings) of the task(s) that elicited their tremor. This ensured that we recorded at least one trial with tremor being present during most of the trial. For each patient, we present results for the trial during which tremor was most persistent.

Surface EMG decomposition. Motor unit spike trains were identified from the multichannel surface EMG with the convolution kernel compensation (CKC) technique (Holobar et al. 2009; Holobar and Zazula 2007) and manually verified by an experienced operator. The CKC technique has been validated with the decomposition of motor neuron activities in more than 15 muscles and 500 healthy subjects performing voluntary contractions (e.g., Holobar et al. 2009, 2010) and has recently been shown to work accurately also for EMG signals of tremor patients (Holobar et al. 2012). Specifically, the decomposition method has been shown to accurately decompose signals with paired and tripled discharges, i.e., firings with an ISI in the $\sim 10-90$ ms range, as observed in pathological tremor (Baker et al. 1992; Christakos et al. 2009; Das Gupta 1963; Dietz et al. 1974; Elek et al. 1991). This technique is also the only one that was proved to be accurate for extremely high levels of motor unit synchronization (Holobar et al. 2012), as may be expected in tremor.

Since EMG decomposition accuracy was fundamental for assessing the properties of the neural drive to muscle and common synaptic inputs in ET patients, we defined two inclusion criteria for the identified single motor unit spike trains. First, given that the estimation of the characteristics of common inputs to motor neurons and the computation of corticospinal coherence typically require a sufficiently large number of epochs, we excluded those motor units that were not firing during $>65 \%$ of the trial. In addition, to ensure that only motor units whose spike trains were identified with great accuracy were considered in the analysis, we computed for each of them the signalto-interference metric proposed in Holobar et al. (2014). This metric assessed the quality of the decomposition by comparing the height of the spike trains identified to the baseline jitter of the CKC algorithm. A threshold of $28 \mathrm{~dB}$ was applied to this metric for the exclusion of motor units whose discharge patterns were not identified with high reliability (Holobar et al. 2014). Figure 1 shows an example of decomposition of the surface EMG.

Data processing and analysis. This section presents the methodology employed to investigate motor unit synchronization, the characteristics of the common synaptic inputs to the motor neuron pool, and how the discharge pattern of individual and groups of motor neurons related to the cortical activity. In some analyses several motor unit spike trains were pooled to build a so-called composite spike train (CST; Farina et al. 2013; Negro and Farina 2011a, 2012). The CST 
Fig. 1. Example of EEG, surface EMG, and gyroscope signals recorded and of a few motor units identified through the decomposition of the multichannel surface EMG. The data correspond to patient 03 . A: recordings from 3 EEG channels. $B$ : signals from all the channels of the 4th column of the surface EMG electrode array (rows 1-12). a.u., Arbitrary unit. $C$ : hand tremor as recorded with a pair of gyroscopes. $D-F$ are related to motor unit discharges. $D$ : shape of the motor unit action potential of 1 of the motor neurons identified, for all the channels of the 4th and 5th columns of the electrode array (rows 1-12). E: spike trains discharged by 5 of the motor units $(\mathrm{mu})$ identified for this patient. $F$ : interspike interval (ISI) histogram of 2 of these motor neurons, exhibiting a clear bimodal pattern caused by the occurrence of paired (or tripled) discharges and the subsequent firings to complete a tremor burst.

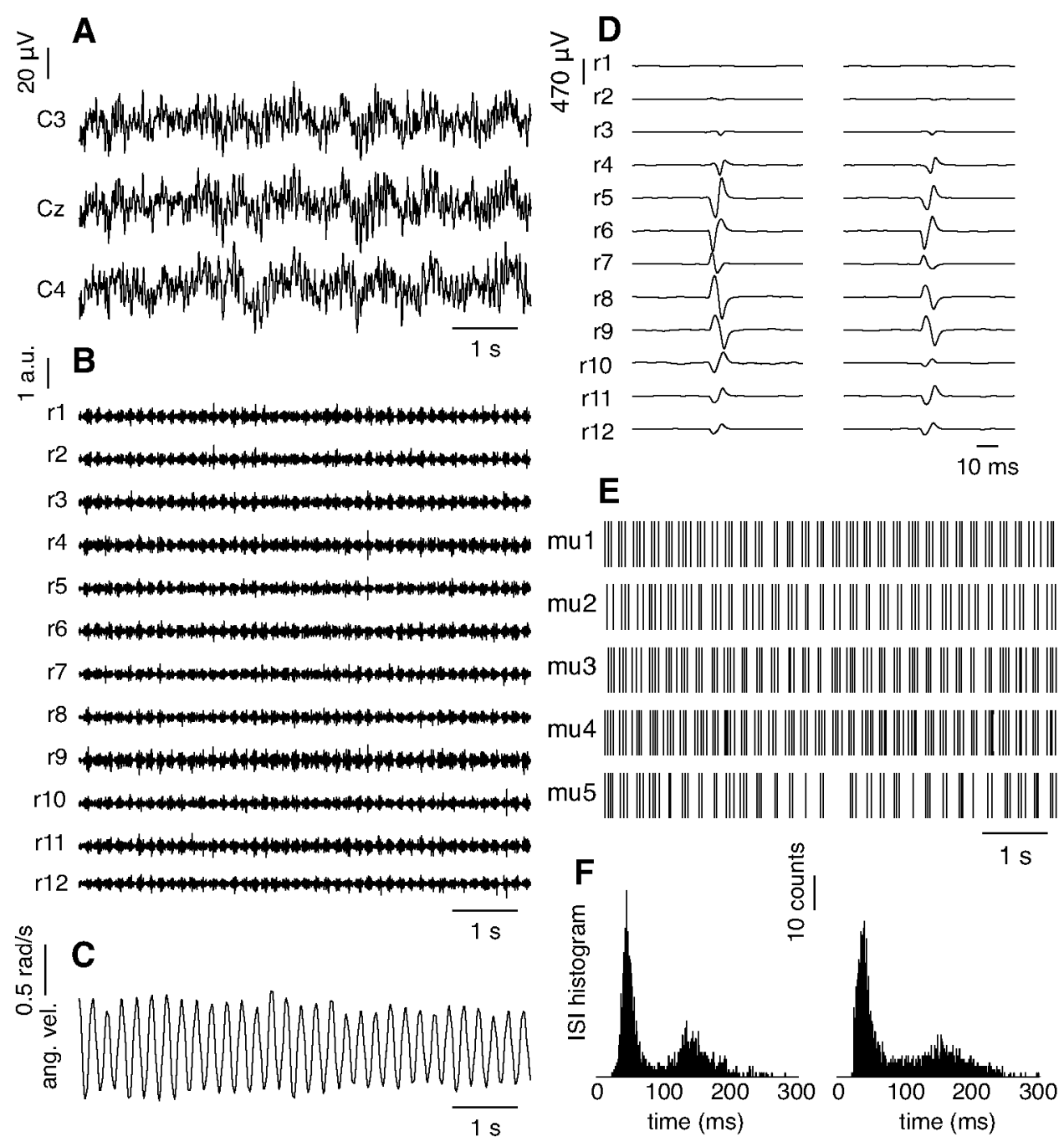

constitutes the best representation of the common synaptic input to motor neurons (Farina et al. 2013, 2014a), which is the neural drive to the muscle (Farina et al. 2014b), and is strongly correlated with muscle force (Negro et al. 2009). EEG channels were spatially filtered with the Hjorth transform (Hjorth 1975) (16 electrodes: Fz, FC3, FC1, $\mathrm{FCz}, \mathrm{FC} 2, \mathrm{FC} 4, \mathrm{C} 3, \mathrm{C} 1, \mathrm{Cz}, \mathrm{C} 2, \mathrm{C} 4, \mathrm{CP} 3, \mathrm{CP} 1, \mathrm{CPz}, \mathrm{CP} 2$, and CP4), and artifacts were carefully removed. Manual inspection, in combination with a threshold (defined as the mean $\pm 3 \mathrm{SD}$ of a signal composed of 20 high-quality 1-s epochs chosen from different parts of the trial), served to ensure that the resultant EEG signal did not contain significant artifacts.

Motor unit synchronization was estimated with a commonly employed technique (Nordstrom et al. 1992), which is based on the computation of cross-correlograms between pairs of motor unit spike trains (Kirkwood and Sears 1978; Nordstrom et al. 1992). To this end, for each trial we calculated the cross-correlation histogram and its correspondent cumulative sum $( \pm 100 \mathrm{~ms}$ relative to the reference motor neuron discharge, in 1-ms bins; normalized by dividing each bin by the mean of the cross-correlation histogram) for all pairs of motor units. The position and duration of the synchronous peak in the cross-correlation histogram considered to be significant were calculated from the cumulative sum (Ellaway 1978), by finding the first relative minimum moving backward from the reference motor neuron discharge and the first relative maximum moving forward (Dideriksen et al. 2009). We considered this cross-correlation peak significant if the relative extrema of the cumulative sum function that identified it were above the mean of the baseline of the cross-correlogram by $>3$
SDs of the first 50 bins (Davey et al. 1986). Finally, the common input strength (CIS) index was computed for all pairs of motor neurons exhibiting significant synchronization as the number of counts within the synchronous peak in excess of that expected by chance divided by the time during which the motor units were active (Nordstrom et al. 1992). The last 2 min of the trial with stable motor unit firings was considered for these calculations, to enable comparison with the literature.

The frequency analysis of the common synaptic inputs to the motor neuron pool was performed by computing, for each trial, the mean coherence between all possible combinations of pairs of CSTs comprising the maximum possible number of different motor units (Negro and Farina 2012). For example, if seven motor units were identified from a muscle, we calculated the coherence function for each possible pair of CSTs comprising four and three different motor unit spike trains and then averaged the coherence for all pairs. This has recently been shown to be the most effective means of characterizing the frequency content and strength of the common inputs to motor neurons (Negro and Farina 2012). Furthermore, we investigated the relationship between motor unit synchronization as computed with the CIS and the common input strength as estimated from the coherence between pairs of CSTs by computing the CIS for the same data windows.

Finally, corticospinal coherence was computed to assess the cortical contribution to the neural drive to muscle, i.e., the CST. This allowed verification of the hypothesis that the central oscillations of ET are a common cortical projection to the motor neuron pool and 
investigation of their role as causative factors of the observed strength of common synaptic input. We calculated the corticospinal coherence between the 16 processed, artifact-free EEG channels and all the possible combinations of CSTs comprising between 1 and the total number of motor units identified during the trial. To test the hypothesis of common cortical projection to the motor neuron pool, we assessed, for the channel exhibiting the largest corticospinal coherence at the tremor frequency, how the coherence varied with the number of motor units considered: if the projection were common to the entire motor neuron pool, the coherence should increase monotonically, reaching a plateau, as more motor neurons were considered in the CST (Gallego et al. 2011; Negro and Farina 2011a).

The coherence functions between motor units and between motor units and EEG were calculated following the method proposed in Halliday et al. (1995). First, the CSTs and/or EEG signals were divided into epochs of 1-s duration, from which the individual power spectra and the cross-spectrum (1-s Hann window, $0.125-\mathrm{Hz}$ resolution, achieved with zero-padding) were computed. Then coherence was calculated as the ratio of the magnitude squared cross-spectrum to the product of their individual power spectra (e.g., Halliday et al. 1995; Hellwig et al. 2001). The confidence limit was obtained as proposed in Rosenberg et al. (1989).

Throughout this report, results are given as means \pm SD. Statistical tests were considered significant if $P<0.05$. Correlations between pairs of variables were investigated with either Pearson's or Spearman's correlation; the latter was employed when the data did not conform to normality (Lilliefors test, $P<0.05$ ). Differences in the strength of common input at different frequency bands were assessed with a Student's paired $t$-test. We tested whether motor unit synchronization as estimated with the CIS was significantly greater than for controls with a Wilcoxon signed-rank test. To calculate the minimum number of motor neurons that most accurately transmitted the tremorrelated cortical activity, we compared the magnitude of the coherence at the tremor frequency for the pooled data of all the patients with a Student's unpaired $t$-test. Pairs of corticospinal coherence estimates obtained for CSTs comprising $n$ and $n+1$ motor units were compared for increasing values of $n$ until a nonsignificant difference was found.

\section{RESULTS}

The total number of identified motor unit spike trains was 56 (6.2 \pm 2.4 motor units per trial; see Table 1 for details). The average motor unit discharge rate over all patients had large variability, ranging from $9.0 \pm 2.9$ to $18.1 \pm 3.9$ pps (Table 1 ). There was no consistent relationship between motor unit firing rate and tremor frequency across patients (Fig. $2 A$ ). However, mean discharge rate was a poor indicator of motor unit prop- erties since the ISI distributions varied among patients and included bimodal distributions. Therefore, we further analyzed the individual ISI histograms for each motor unit. The ISI histogram of the motor units discharges (Fig. 2B) followed either 1) a bimodal distribution (patients 01-04), with the first peak corresponding to paired or tripled discharges (average position of the peak $34.6 \pm 9.1 \mathrm{~ms}$ ) and a second peak associated to the tremor frequency (average position, $190.5 \pm$ $45.0 \mathrm{~ms}$; see the representative examples in Fig. $1 F$ and Fig. $2 B$ ), or 2) a unimodal distribution (patients 05-09; average position of the peak $67.3 \pm 26.2 \mathrm{~ms}$ ), with a peak not significantly correlated with the tremor frequency $(P=0.100$, Spearman's correlation). The ISI histograms in Fig. 2 were built with all motor units together for each patient since all the units within a patient showed the same distribution of ISI. From Fig. $2 A$ it is evident that there was no difference between the tremor frequency of the patients showing the two types of ISI distributions (range 4.8-6.1 $\mathrm{Hz}$ and 4.9-6.6 $\mathrm{Hz}$, respectively). Finally, the relative proportion of paired and tripled discharges (range $37.43-68.15 \%$ for those patients with a bimodal ISI histogram) varied considerably among motor units and patients, as reported for patients with Parkinson's disease (Christakos et al. 2009; Dietz et al. 1974).

Motor unit synchronization. The analysis of cross-histograms of motor unit spike train pairs indicated that the activities of 132 of 169 motor unit pairs $(78.1 \%)$ were significantly synchronized. The average CIS over all motor units of all patients was $1.44 \pm 1.44 \mathrm{pps}$ (see values per patient in Table $1)$, an average value significantly greater $(P<0.001$, Wilcoxon signed-rank test) than that reported for healthy subjects for the same muscle group during voluntary contractions (mean value $\leq 0.7$ pps; Keen and Fuglevand 2004; Schmied et al. 1993). The CIS value was not associated to the tremor frequency ( $P=0.472$, Pearson's correlation).

Sources of common inputs to motor neurons. Figure 3 shows the coherence analysis between pooled motor unit spike trains for each patient. In all cases there were two large peaks in the coherence spectrum, which indicated the presence of two main sources of common input to the motor neuron pool: one at low frequency $(<2-3 \mathrm{~Hz})$, presumably related to the voluntary common drive to muscle (De Luca and Erim 1994; Negro et al. 2009; Negro and Farina 2012), and a second peak at the tremor frequency (mean frequency $5.5 \pm 0.9 \mathrm{~Hz}$, indicated with

Table 1. Summary of motor neuron synchronization and corticospinal coherence

\begin{tabular}{|c|c|c|c|c|c|c|c|c|c|}
\hline Type of tremor & PO & $\mathrm{RE}$ & $\mathrm{PO}$ & $\mathrm{PO}$ & $\mathrm{PO}$ & $\mathrm{PO}$ & $\mathrm{PO}$ & PO & $\mathrm{PO}$ \\
\hline No. of MUs & 5 & 5 & 11 & 4 & 5 & 4 & 9 & 7 & 6 \\
\hline CIS [2 min], pps & $1.45 \pm 0.22$ & $0.98 \pm 1.47$ & $2.32 \pm 1.96$ & $0.95 \pm 0.76$ & $1.61 \pm 0.48$ & $1.63 \pm 1.00$ & $1.46 \pm 1.27$ & $0.36 \pm 0.53$ & $0.96 \pm 0.92$ \\
\hline EEG channel & $\mathrm{C} 3$ & $\mathrm{C} 1$ & $\mathrm{FC} 4$ & $\mathrm{FC} 3$ & $\mathrm{CP} 3$ & $\mathrm{CP} 4$ & $\mathrm{FC} 2$ & CP3 & $\mathrm{CP} 2$ \\
\hline Freq. beta, $\mathrm{Hz}$ & 27.3 & 12.4 & 29.3 & 12.4 & 26.9 & 15.3 & n.s. & 20.1 & 17.6 \\
\hline
\end{tabular}

Table shows, for each patient, the type of tremor elicited [postural (PO) or rest (RE)], the number of motor units (MUs) identified through the decomposition of the surface EMG, the grand mean $( \pm \mathrm{SD})$ of their discharge rate, the degree of MU synchronization according to the common input strength (CIS; the last 2 variables were computed in 2-min windows), the EEG channel that exhibited the largest coherence at the tremor frequency, and the magnitude and frequency at which the coherence peaks at the tremor frequency and the beta band were found. All coherence values reported were statistically significant $(P<0.05)$, except where otherwise noted (n.s.). 

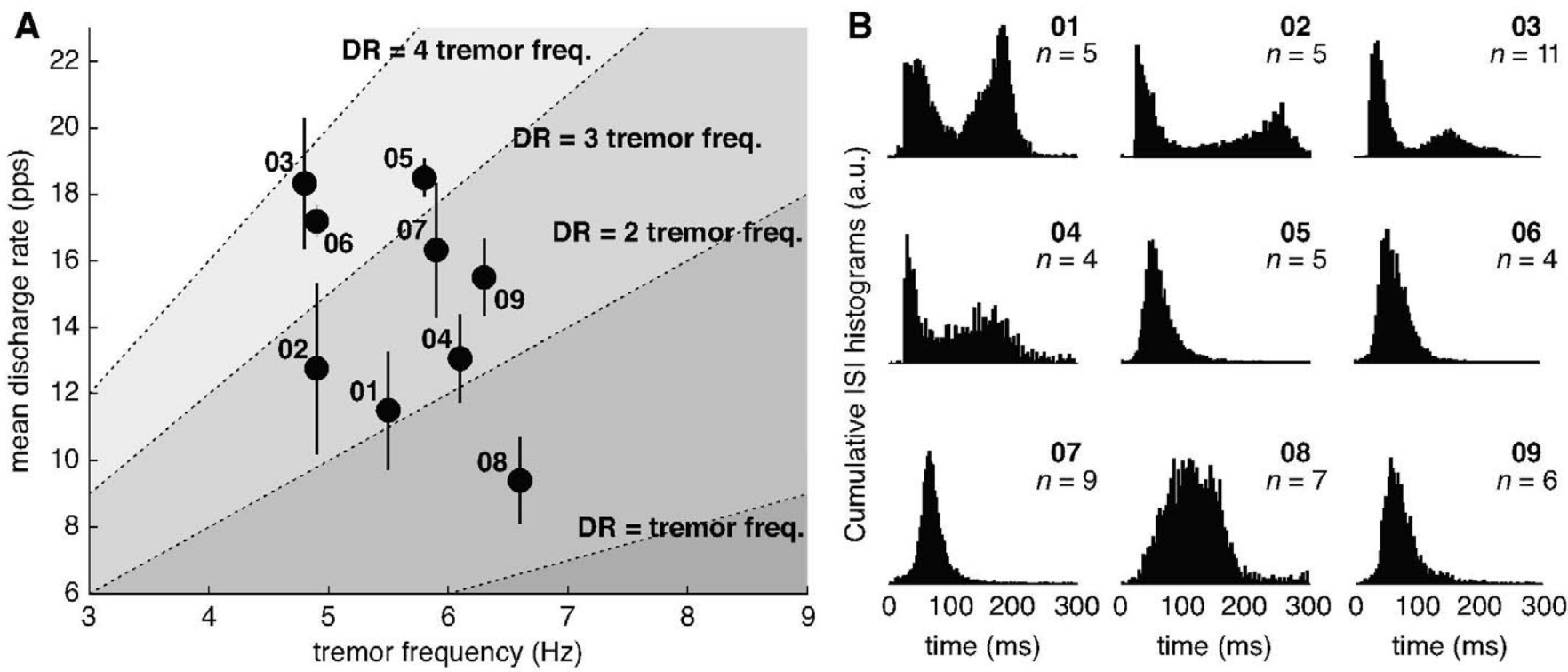

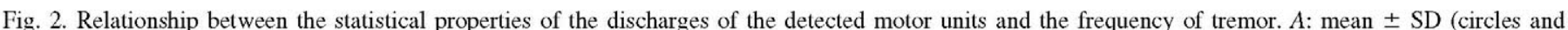

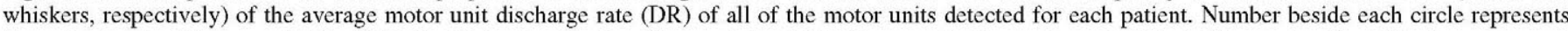

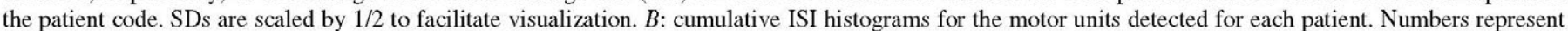

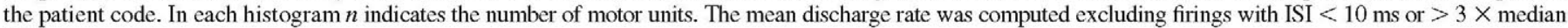
(ISI).

arrows in Fig. 3). This suggests that, in addition to the common drive that reflected the neural control of voluntary contractions ( 8 of 9 patients were holding their hands outstretched), the motor neuron pool received common input at the tremor frequency. The extents to which both common synaptic inputs were shared across the motor neurons (i.e., coherence values at the 2 frequencies) were independent of each other $(P=0.795$, Pearson's correlation), the coherence at the tremor frequency being significantly greater $(P=0.002$, Student's paired $t$-test $)$. These common inputs may reflect not only the descending drive to muscle but also the contribution of spinal afferents (Dartnall et al. 2008; Farina et al. 2010). The coherence spectra of patients 01,02 , and 08 also exhibited clear peaks at frequencies that were harmonics of that of the tremor (Fig. 3). Because two of these patients (patients 01 and 02) had a bimodal ISI histogram contrary to patient 08 (Fig. 2), these coherence peaks were not associated to the type of ISI distribution.

Direct examination of the motor unit spike trains explains the high coherence at the tremor frequency. Figure 4 shows the filtered motor unit spike trains (band pass, 3-10 Hz, zero phase), which in the tremor band are oscillations at the same frequency and phase as the tremor oscillations. The similarity of these oscillations among motor units indicates the common nature of the generating input.

Finally, the mean coherence value between CSTs at the tremor frequency was significantly correlated with the mean CIS (calculated with the same data windows; see Fig. 3) across patients ( $P=0.005, r=0.840$, Pearson's correlation).

Corticospinal coupling. The average number of 1 -s epochs per subject not influenced by EEG artifacts, and with stable discharges of the identified motor neurons, was $97.4 \pm 50.6$ (range 36-182). These were the data used in the calculations of corticospinal coupling.

Figure 5 displays an example of corticospinal coherence as estimated from the motor unit activities and the processed EEG signal recorded at the contralateral sensorimotor cortex (where the largest coherence was found, as expected). The plots of coherence correspond to the functions obtained when varying the number of motor unit spike trains used for the calculation (from 1 to 11 in this example). The coherence peak at the tremor frequency $(\sim 4.75 \mathrm{~Hz}$, indicated by black arrow in Fig. $5 A$ ) was above confidence level for any number of motor units, even when using only one unit, indicating a strong tremorrelated cortical projection. Moreover, the magnitude of the corticospinal coherence at the tremor frequency increased monotonically with the number of motor neurons considered, until a plateau was reached when approximately five motor neurons were included in the CST (Fig. 5B). Considering more than five motor units for the estimate negligibly increased the amount of coherence (for example, the increase when considering 6 motor units was $0.5 \%$ with respect to 5 , and when considering 11 it was $1.5 \%$ with respect to 5 ). The estimation of corticospinal coherence was relatively invariant to which motor units were chosen to build the CST, as shown by the small SD of the values in Fig. $5 B$. These observations verify the hypothesis that the descending tremor-related cortical activity was common to the entire motor neuron pool (Gallego et al. 2011; Negro and Farina 2011a). The coherence spectra also showed a significant peak at the beta band (indicated by gray arrow in Fig. 5A), which is related to voluntary descending commands (e.g., Conway et al. 1995; Negro and Farina 2011a). The coherence in this band also increased monotonically as more motor units were considered, but the trend was slower and the values had greater SD than for the coherence at the tremor band (Fig. 5B). Therefore, for this patient differences existed in the manner in which both descending drives were projected to the output of the motor neuron pool. As expected, the frequency of the hand oscillations corresponded to the tremor frequency peak of the corticospinal coherence (indicated by black arrow in Fig. 5, $A$ and $C$ ). 

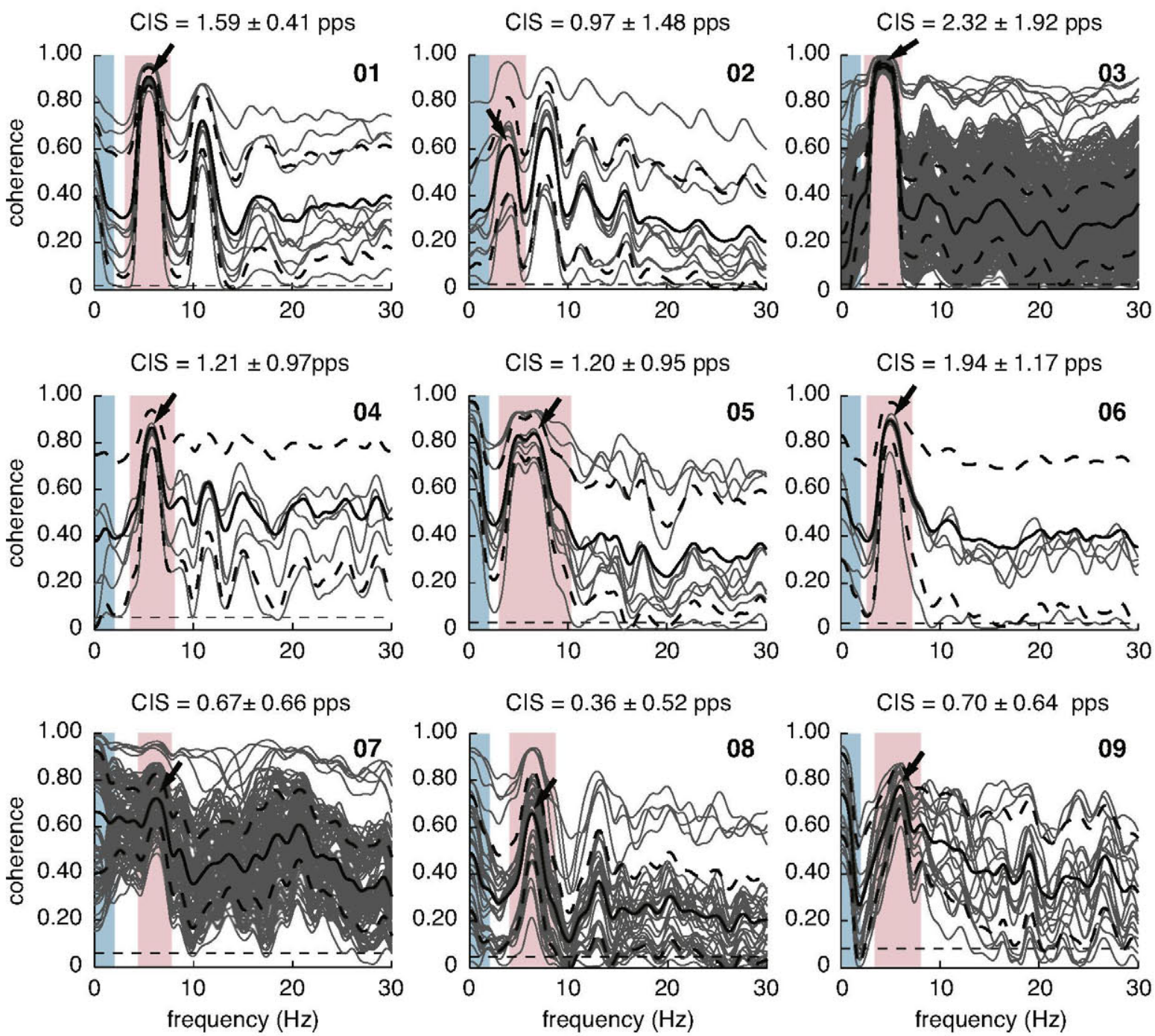

Fig. 3. Estimation of common synaptic inputs to the motor neurons identified, for all patients. Plots show the coherence spectra for all possible pairs of composite spike trains (CSTs) each comprising the largest possible amount of motor unit spike trains (in gray), with their mean (solid black trace) \pm SD (dashed black trace). Each panel represents a single patient. Numbers represent the patient code. The frequency bands that correspond to the common voluntary drive and the common input at the tremor frequency are shaded in blue and red, respectively. The mean $\pm \mathrm{SD}$ common input strengths (CISs) for the same data window that were employed to compute the coherence between pairs of CSTs are displayed at top of each plot.

Similar results were obtained for all the patients analyzed (Table 1). In all cases, the corticospinal coherence function showed a significant peak at the tremor frequency and, in eight patients, another peak in the beta band. Significant coherence at the beta band was found even in the patient who performed the rest task (patient 02; see Table 1), which implies that also in this case there was a certain amount of voluntary descending command. The only patient who did not show significant corticospinal coherence in the beta band was the patient with the greatest number of signal epochs excluded because of artifacts. The relatively small number of epochs (49) used for the computation of coherence may have been insufficient to identify a significant coherence level at high frequencies. Finally, it is worth observing that, although always above the confidence level, the corticospinal coherence values at the tremor frequency were relatively small (Fig. 5 and Table 1).

As observed for patient 03 (Fig. $5 B$ ), in all the patients the corticospinal coherence at the tremor frequency increased monotonically as more motor units were included in the CST, and concurrently the variability of the estimate decreased (Fig. $6 A$ ). Moreover, in all patients, the coherence values tended to a maximum when a relatively small number of units was used (Fig. 6A). Statistical analysis of the pooled data of all patients indicated that five motor units $(P<0.05$, Student's unpaired $t$-test) resulted in an accurate transmission of the corticospinal input, i.e., the increase in corticospinal coherence was negligible after five motor units were used for the estimate. As mentioned above, this indicates that tremor was a common cortical projection to the motor neuron pool (Negro and Farina 2011a). Interestingly, for seven patients (all except patients 02 and 08 ) the estimation of corticospinal coherence with only one motor unit showed a peak at the tremor frequency above the confidence level, as for the representative case of Fig. 5. This indicated that in most cases the descending cortical tremor input was sufficiently strong that it could even be observed in the output of a single motor neuron.

The magnitude of the coherence in the beta band increased monotonically with the number of units for all patients, as for 

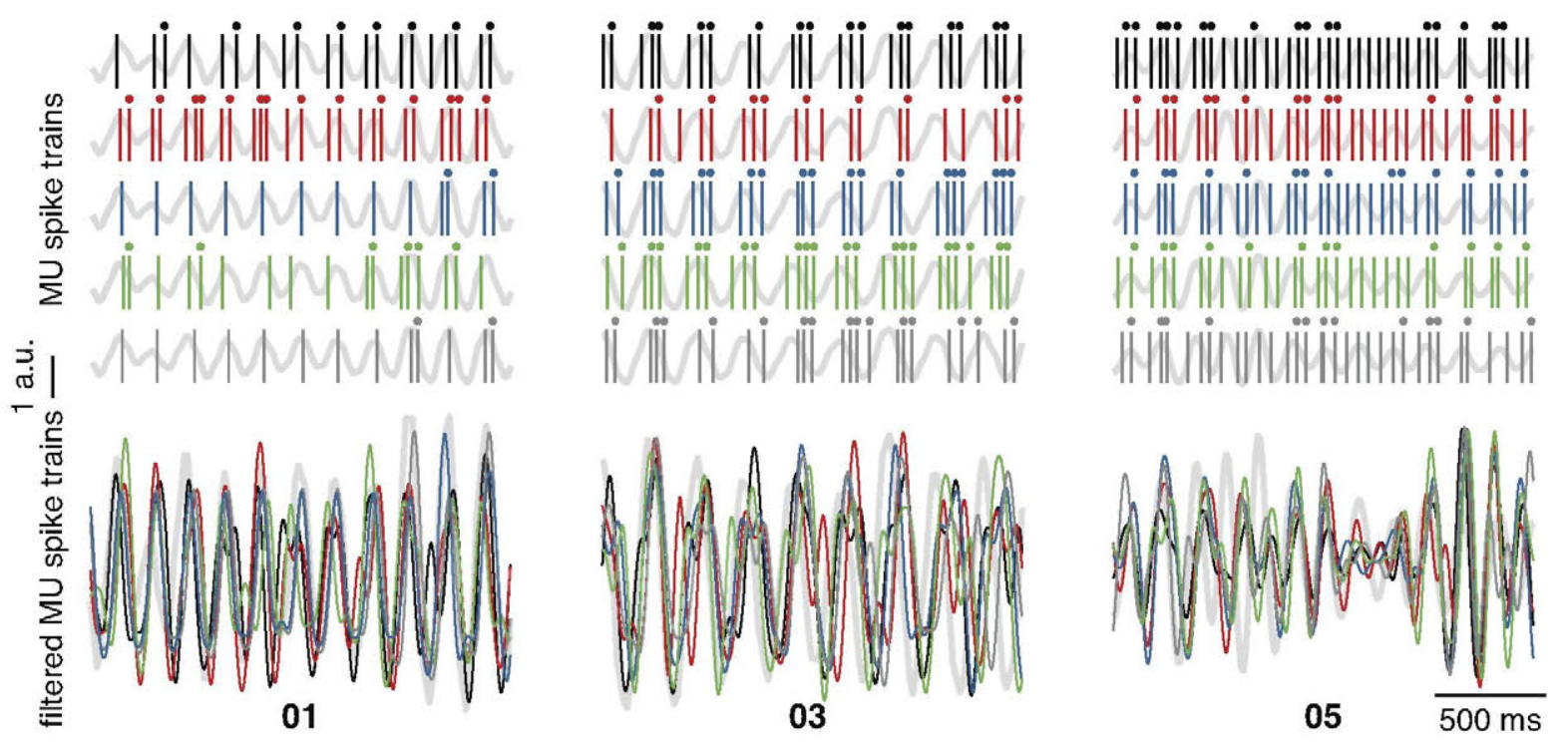

Fig. 4. Examples of motor unit (MU) spike trains for 3 patients. For each patient, the firings of 5 motor units randomly chosen among those identified and their filtered version (band pass, 3-10 Hz, zero phase) are shown at top and bottom (displayed in the same color), respectively. Both are compared to the hand motion (light gray traces in the background) to emphasize how the motor units encode the tremor. Paired discharges are marked with a dot on top of the discharge. Numbers represent the patient code. Figure illustrates the observed large motor unit synchronization and how motor unit firing patterns sometimes fluctuate (see patient 05).

the tremor frequency, but did not reach a constant value with the maximum number of detected units in seven patients.

Finally, we found no significant relation between the magnitude of the corticospinal coherence at the tremor frequency and the corresponding peak in the coherence between CSTs $(P=0.445$, Pearson's correlation).

\section{DISCUSSION}

We have systematically investigated the characteristics of the motor unit spike trains in ET patients and the sources of common synaptic input that the motor neurons receive. This analysis was possible because of a recently developed method of decomposition of multichannel surface EMG recordings
(Holobar et al. 2012) that circumvents the methodological limitations of traditional approaches using intramuscular electrodes (Merletti and Farina 2009). This study demonstrates, for the first time, that the motor units in ET patients exhibit a greater degree of synchronization than those in healthy individuals, which implies the existence of strong common synaptic inputs to the motor neuron pool. The high level of common input to motor neurons was confirmed by the analysis of coherence between CSTs, which showed that the increase in synchronization occurs mainly because of a common input at the tremor frequency. Corticospinal coupling, studied between EEG and CSTs, indicated that the tremor-related cortical activity is a common projection to the motor neuron pool.
Fig. 5. Example of coherence between EEG signals recorded at the contralateral sensorimotor cortex (FC4, given that we recorded the left hand) and the CSTs. Data are from patient 03. A: average coherence for all possible CSTs comprising from 1 to 11 motor neurons (black solid lines). These coherence spectra always exhibited a significant peak at tremor frequency (black arrow), whose height increased monotonically with the number of motor units considered. Coherence at the beta band (gray arrow), corresponding to the voluntary drive to muscle, became significant when 7 motor units were included in the CST. The confidence level $(P<0.05)$ is represented as a dashed black line. $B:$ mean $\pm \mathrm{SD}$ (circle and length of whiskers, respectively) of the coherence at tremor frequency (in black; corresponds to peak indicated by black arrow in $A$ ) and the beta band (in gray; corresponds to the peak indicated by gray arrow in $A$ ) as a function of the number of motor units in the CST. $C$ : amplitude spectrum of the hand tremor as recorded with the solid-state gyroscopes, showing a clear peak at tremor frequency (black arrow), which appeared very close to that observed in the coherence plots depicted in $A$. $D$ : hand oscillations during a portion of the trial.
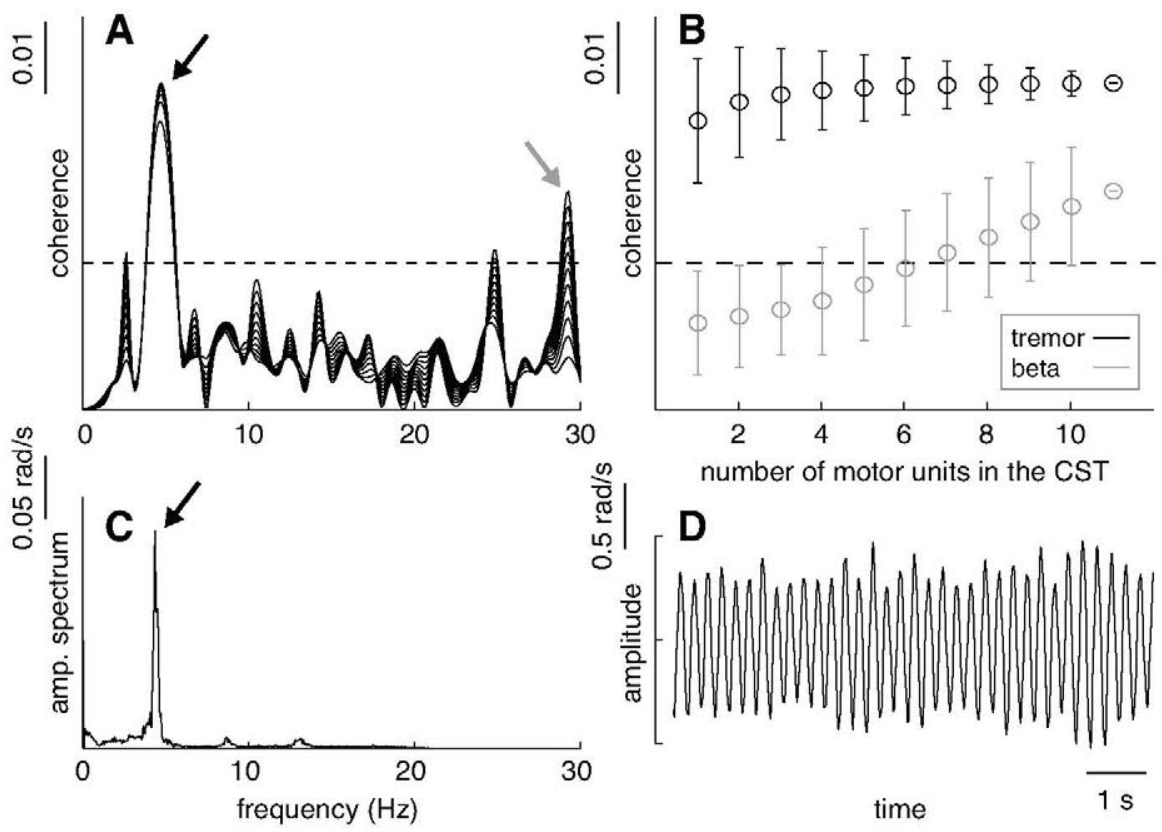

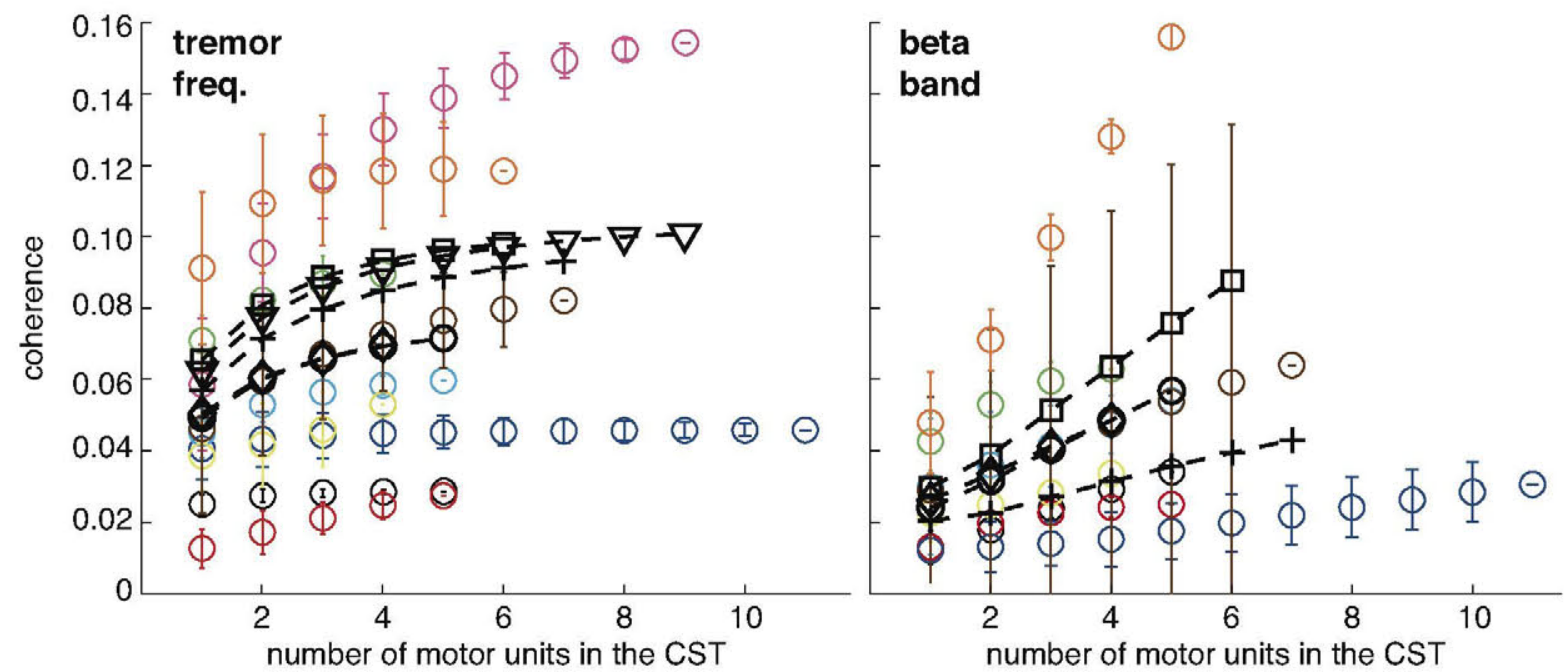

Fig. 6. Estimation of corticospinal coherence at the tremor frequency (left $t)$ and the beta band $(r i g h t)$ as a function of the number of motor units considered, for all patients. Circles and whiskers represent mean \pm SD of the coherence peak at the selected frequency, obtained for all the possible combinations of motor units to form a CST. Results are shown as a function of the number of motor units included in the calculations, and each patient is represented in a different color. Patients are codified as follows: patient 01, black; patient 02, red; patient 03, blue; patient 04, green; patient 05, cyan; patient 06, yellow; patient 07, magenta; patient 08 , brown; patient 09 , orange. A series of grand means are also displayed (thick black lines) to represent the general trend of the data: for all patients $(\diamond)$, for all patients with 5 or more motor units detected $(0)$, for all patients with 6 or more motor units detected ( $\square$ ), for all patients with 7 or more motor units detected $(+)$, and for all patients with 9 or more motor units detected $(\nabla)$.

Despite the relative similarity in the mechanical manifestation of tremor among patients, the underlying motor unit discharges had different statistical distributions (see Fig. 2). Nonetheless, the properties of the common input were consistent across patients as revealed by the analysis of coherence between CSTs that showed two main inputs for all patients. Since the degree of motor unit synchronization was correlated to the coherence value at the tremor frequency, synchronization among motor units was increased by the common synaptic input at the tremor frequency (Kirkwood and Sears 1978; Nordstrom et al. 1992; Sears and Stagg 1976). Indeed, motor unit synchronization provides an estimate of the global strength of synaptic input for the entire frequency bandwidth, whereas coherence shows synchronization for each frequency (Negro and Farina 2012). The data presented provide the first experimental proof of high synchronization levels among motor units in ET patients and show that high synchronization occurs specifically with an oscillation at the tremor frequency, thus causing rhythmic entrainment that contributes to the generation of tremor. This association has been hypothesized previously (e.g., Elble and Deuschl 2009; Elek et al. 1991; McAuley and Marsden 2000) but never proven directly. Given the evidence that motor unit synchronization does not differ between young and old adults (Kamen and Roy 2000; Semmler et al. 2000), we conclude that this greater than normal synchronization was caused by the tremor input to motor neurons, and was not an effect of age.

Previous studies reported that the cortical oscillations causing ET are projected to tremulous muscles through the corticospinal tract, based on the observation of significant coherence at the tremor frequency between EEG and EMG recordings (Hellwig et al. 2001, 2003; Muthuraman et al. 2012; Raethjen et al. 2007). We reanalyzed these observations by computing the coherence between EEG and motor unit spike trains. With our analysis at the single-motor unit level, we also found significant corticospinal coherence between the con- tralateral sensorimotor cortex and motor unit cumulative spike train (CST; see Table 1), confirming the studies based on the interference EMG. Despite the agreement in conclusions based on EEG-EMG coherence and our EEG-motor unit coherence data, we showed the association between EEG and motor neuron output directly, which is stronger evidence of a direct influence of the corticospinal tract in tremor generation (Negro and Farina 2011a). Furthermore, we also studied the behavior of coherence with EEG when the number of motor unit spike trains considered was progressively increased. This analysis showed that as more motor unit spike trains were analyzed the coherence at the tremor frequency increased monotonically, up to a constant value reached for approximately five motor units, and the variability of coherence estimates decreased (Fig. 6A). These observations indicate not only the presence of corticomuscular coupling but also that the central oscillations causing ET are a common projection to the entire motor neuron pool (Gallego et al. 2011; Negro and Farina 2011a). On the basis of the present results, it is unlikely that intermittent nonlinear corticomuscular interaction participates in the transmission of the central oscillations that cause ET, as proposed by Raethjen et al. (2007).

Concurrently with the presence of significant corticospinal coupling at the tremor frequency, we also observed significant coherence between the EEG and motor unit spike trains in the beta band. This is assumed to represent the voluntary drive sent to motor neurons by the corticospinal tract (e.g., Conway et al. 1995; Negro and Farina 2011a). Therefore, in ET patients the motor neuron pool concurrently samples two strong common inputs with different frequency content, which likely facilitates the occurrence of tremor during the performance of voluntary movements (e.g., Benito-León and Louis 2006; Deuschl et al. 2000). Notably, both common synaptic inputs are also observed directly from the analysis of coherence between CSTs at the spinal level (see Fig. 3). 
Since the strength of corticospinal coupling at the tremor frequency was uncorrelated with the magnitude of the coherence between CSTs (which represents the net common synaptic input) at the same frequency, it is unlikely that the cortical input was the only source of common input to motor neurons at the tremor frequency. Accordingly, the corticospinal coherence values were very low, as in previous work (Raethjen et al. 2007), which indicates the presence of additional sources of common input at the tremor frequency that may decrease the correlation with the common cortical input (Negro and Farina 2011b). We therefore hypothesize that the afferent component, which is projected to the entire motor neuron pool by Ia fibers (Mendell and Henneman 1971), or additional supraspinal descending drives provide a substantial contribution to the common input received by motor neurons at the tremor frequency. The potential role of the afferent input, in particular, is in agreement with evidence showing that modification of the mechanical properties of the tremulous limb alters the tremor in ET (e.g., Elble et al. 1987; Héroux et al. 2009). Moreover, the observation that in some cases there were significant peaks at the first tremor frequency harmonic in the coherence between CSTs (Fig. 3), while these peaks were never observed in the EEG-CST coherence (see example in Fig. 5), indicates that they were likely generated by common projections of afferent pathways because of their resonant behavior. The hypothesis that muscle spindles contribute significantly to the generation of the tremor in ET could be further investigated by experiments manipulating the level of afferent activity. For example, reduction of Ia activity by means of localized ischemia (Allum and Mauritz 1984; Sinkjaer and Hayashi 1989) or by the restriction of limb movement (isometric conditions) could be applied and the effect on the neural drive to the muscle and the corticospinal coherence could be assessed.

In conclusion, this study systematically analyzed for the first time the neural drive to muscle in ET patients with a novel noninvasive approach that offers a unique view into the output of the spinal cord circuitries in vivo. We demonstrated that motor units in ET are highly synchronized because of the presence of strong common synaptic input to motor neurons at the tremor frequency. This common input is partly corticospinal, as shown by the analysis of coherence between EEG and motor unit spike trains. However, it is weakly associated with the net common input at the tremor frequency (coherence between CSTs), which indicates a contribution of common input from spinal or secondary supraspinal sources. These data are the first that provide a complete description of the characteristics of motor unit spike trains in ET.

\section{ACKNOWLEDGMENTS}

The authors gratefully thank Dr. Juan Manuel Belda-Lois (Instituto de Biomecánica de Valencia, Valencia, Spain) and Dr. Ascensión Castillo (Hospital General de Valencia, Valencia, Spain) for their help with the experiments in Valencia and Juan Pablo Romero, Dr. Ignacio Javier Posada-Rodríguez, Dr. Félix Bermejo-Pareja, and Dr. Julián Benito-León (University Hospital "12 de Octubre," Madrid, Spain) for their help with the experiments in Madrid.

\section{GRANTS}

This work was funded by the EU Commission [grant no. EU-FP7-2011287739 (NeuroTREMOR)].

\section{DISCLOSURES}

No conflicts of interest, financial or otherwise, are declared by the author(s).

\section{AUTHOR CONTRIBUTIONS}

Author contributions: J.A.G., J.L.D., A.H., E.R., and D.F. conception and design of research; J.A.G., A.H., J.I., and E.R. performed experiments; J.A.G., J.L.D., A.H., and J.I. analyzed data; J.A.G., J.L.D., A.H., J.I., E.D.L., E.R., and D.F. interpreted results of experiments; J.A.G. prepared figures; J.A.G., J.L.D., and D.F. drafted manuscript; J.A.G., J.L.D., A.H., J.I., J.L.P., E.D.L., E.R., and D.F. edited and revised manuscript; J.A.G., J.L.D., A.H., J.I., J.L.P., E.D.L., E.R., and D.F. approved final version of manuscript.

\section{REFERENCES}

Allum J, Mauritz K. Compensation for intrinsic muscle stiffness by shortlatency reflexes in human triceps surae muscles. J Neurophysiol 52: 797$818,1984$.

Baker JR, Davey NJ, Ellaway PH, Friedland CL. Short-term synchrony of motor unit discharge during weak isometric contraction in Parkinson's disease. Brain 115: 137-154, 1992.

Benito-León J, Louis ED. Essential tremor: emerging views of a common disorder. Nat Clin Pract Neurol 2: 666-678, 2006.

Boonstra TW, Roerdink M, Daffertshofer A, van Vugt B, van Werven G, Beek PJ. Low-alcohol doses reduce common 10- to $15-\mathrm{Hz}$ input to bilateral leg muscles during quiet standing. I Neurophysiol 100: 2158-2164, 2008.

Christakos CN, Erimaki S, Anagnostou E, Anastasopoulos D. Tremorrelated motor unit firing in Parkinson's disease: implications for tremor genesis. J Physiol 587: 4811-4827, 2009.

Conway BA, Halliday DM, Farmer SF, Shahani U, Maas P, Weir AI, Rosenberg JR. Synchronization between motor cortex and spinal motoneuronal pool during the performance of a maintained motor task in man. $J$ Physiol 489: 917-924, 1995.

Dartmall TJ, Nordstrom MA, Semmler JG. Motor unit synchronization is increased in biceps brachii after exercise-induced damage to elbow flexor muscles. J Neurophysiol 99: 1008-1019, 2008.

Das Gupta A. Paired response of motor units during voluntary contraction in Parkinsonism. I Neurol Neurosurg Psychiatry 26: 265-268, 1963.

Davey N, Ellaway P, Stein R. Statistical limits for detecting change in the cumulative sum derivative of the peristimulus time histogram. $J$ Neurosci Methods 17: 153-166, 1986.

De Luca CJ, Erim Z. Common drive of motor units in regulation of muscle force. Trends Neurosci 17: 299-305, 1994.

Deuschl G, Bain P, Brin M. Consensus statement of the Movement Disorder Society on Tremor. Ad Hoc Scientific Committee. Mov Disord 13, Suppl 3: 2-23, 1998.

Deuschl G, Raethjen J, Lindemann M, Krack P. The pathophysiology of tremor. Muscle Nerve 24: 716-735, 2001.

Deuschl G, Wenzelburger R, Löffler K, Raethjen J, Stolze H. Essential tremor and cerebellar dysfunction. Clinical and kinematic analysis of intention tremor. Brain 123: 1568-1580, 2000.

Dideriksen JL, Falla D, Baekgaard M, Mogensen ML, Steimle KL, Farina D. Comparison between the degree of motor unit short-term synchronization and recurrence quantification analysis of the surface EMG in two human muscles. Clin Neurophysiol 120: 2086-2092, 2009.

Dietz V, Hillesheimer W, Freund H. Correlation between tremor, voluntary contraction, and firing pattern of motor units in Parkinson's disease. $J$ Neurol Neurosurg Psychiatry 37: 927-937, 1974.

Elble RJ, Deuschl G. An update on essential tremor. Curr Neurol Neurosci Rep 9: 273-277, 2009

Elble RJ, Higgins C, Moody C.J. Stretch reflex oscillations and essential tremor. J Neurol Neurosurg Psychiatry 50: 691-698, 1987.

Elek JM, Dengler R, Konstanzer A, Hesse S, Wolf W. Mechanical implications of paired motor unit discharges in pathological and voluntary tremor. Electroencephalogr Clin Neurophysiol 81: 279-283, 1991.

Ellaway P. Cumulative sum technique and its application to the analysis of peristimulus time histograms. Electroencephalogr Clin Neurophysiol 45: 302-304, 1978.

Farina D, Holobar A, Merletti R, Enoka RM. Decoding the neural drive to muscles from the surface electromyogram. Clin Neurophysiol 121: 1616$1623,2010$.

Farina D, Negro F, Jiang N. Identification of common synaptic input to motor neurons from the rectified electromyogram. I Physiol 591: 2403-2418, 2013. 
Farina D, Negro F, Jiang N. Reply from Dario Farina, Francesco Negro and Ning Jiang. J Physiol 592: 251-252, 2014a.

Farina D, Negro F, Dideriksen JL. The effective neural drive to muscles is the common synaptic input to motor neurons. J Physiol 592: 3427-3441, $2014 b$.

Farmer SF, Swash M, Ingram DA, Stephens JA. Changes in motor unit synchronization following central nervous lesions in man. $J$ Physiol 463: 83-105, 1993.

Gallego JA, Dideriksen JL, Farina D, Rocon E, Holobar A, Pons JL. A modelling study on the transmission of the central oscillator in tremor by a motor neuron pool. Conf Proc IEEE Eng Med Biol Soc 2037-2040, 2011.

Gallego JA, Rocon E, Roa JO, Moreno JC, Pons JL. Real-time estimation of pathological tremor parameters from gyroscope data. Sensors (Basel) 10: $2129-2149,2010$.

Halliday DM, Rosenberg JR, Amjad AM, Breeze P, Conway BA, Farmer SF. A framework for the analysis of mixed time series/point process data-theory and application to the study of physiological tremor, single motor unit discharges and electromyograms. Prog Biophys Mol Biol 64: 237-278, 1995.

Heckman CJ, Enoka RM. Motor unit. Compr Physiol 2: 2629-2682, 2012.

Hellwig B, Häußler S, Schelter B, Lauk M, Guschlbauer B, Timmer J, Lücking CH. Tremor-correlated cortical activity in essential tremor. Lancet 357: $519-523,2001$.

Hellwig B, Schelter B, Guschlbauer B, Tïmmer J, Lücking C. Dynamic synchronisation of central oscillators in essential tremor. Clin Neurophysiol 114: 1462-1467, 2003.

Héroux ME, Pari G, Norman KE. The effect of inertial loading on wrist postural tremor in essential tremor. Clin Neurophysiol 120: 1020-1029, 2009.

Hjorth B. An on-line transformation of EEG scalp potentials into orthogonal source derivations. Electroencephalogr Clin Neurophysiol 39: 526-530, 1975.

Holobar A, Farina D, Gazzoni M, Merletti R, Zazula D. Estimating motor unit discharge patterns from high-density surface electromyogram. Clin Neurophysiol 120: 551-562, 2009.

Holobar A, Glaser V, Gallego JA, Dideriksen JL, Farina D. Non-invasive characterization of motor unit behavior in pathological tremor. $J$ Neural Eng 9: 056011, 2012

Holobar A, Minetto MA, Botter A, Negro F, Farina D. Experimental analysis of accuracy in the identification of motor unit spike trains from high-density surface EMG. IEEE Trans Neural Syst Rehabil Eng 18: 221-229, 2010.

Holobar A, Minetto MA, Farina D. Accurate identification of motor unit discharge patterns from high-density surface EMG and validation with a novel signal-based performance metric. J Neural Eng 11: 016008, 2014.

Holobar A, Zazula D. Multichannel blind source separation using convolution kernel compensation. IEEE Trans Signal Process 55: 4487-4496, 2007.

Kamen G, Roy A. Motor unit synchronization in young and elderly adults. Eur J Appl Physiol 81: 403-410, 2000.

Keen DA, Fuglevand AJ. Common input to motor neurons innervating the same and different compartments of the human extensor digitorum muscle. J Neurophysiol 91: 57-62, 2004
Kirkwood P, Sears TA. The synaptic connections to intercostal motoneurones as revealed by the average common excitation potential. $J$ Physiol 275: 103-134, 1978.

Kudina LP, Andreeva RE. Motoneuron double discharges: only one or two different entities? Front Cell Neurosci 7: 75, 2013.

Louis ED, Babij R, Cortés E, Vonsattel JP, Faust PL. The inferior olivary nucleus: a postmortem study of essential tremor cases versus controls. Mov Disord 28: 779-786, 2013.

McAuley JH, Marsden CD. Physiological and pathological tremors and rhythmic central motor control. Brain 123: 1545-1567, 2000.

Mendell LM, Henneman E. Terminals of single Ia fibers: location, density, and distribution within a pool of 300 homonymous motoneurons. J Neurophysiol 34: 171-187, 1971.

Merletti R, Farina D. Analysis of intramuscular electromyogram signals. Philos Trans A Math Phys Eng Sci 367: 357-368, 2009.

Muthuraman M, Heute U, Arning K, Anwar AR, Elble R, Deuschl G, Raethjen J. Oscillating central motor networks in pathological tremors and voluntary movements. What makes the difference? Neuroimage 60: 13311339, 2012.

Negro F, Farina D. Linear transmission of cortical oscillations to the neural drive to muscles is mediated by common projections to populations of motoneurons in humans. J Physiol 589: 629-637, 2011a.

Negro F, Farina D. Decorrelation of cortical inputs and motoneuron pool. $J$ Neurophysiol 106: 2688-2697, 2011b.

Negro F, Farina D. Factors influencing the estimates of correlation between motor unit activities in humans. PLoS One 7: e44894, 2012

Negro F, Holobar A, Farina D. Fluctuations in isometric muscle force can be described by one linear projection of low-frequency components of motor unit discharge rates. J Physiol 587: 5925-5938, 2009.

Nordstrom M, Fuglevand AJ, Enoka RM. Estimating the strength of common input to human motoneurons from the cross-correlogram. $J$ Physiol 453: 547-574, 1992.

Raethjen J, Govindan RB, Kopper F, Muthuraman M, Deuschl G. Cortical involvement in the generation of essential tremor. J Neurophysiol 97: $3219-3228,2007$.

Rocon E, Andrade AO, Pons JL, Kyberd P, Nasuto SJ. Empirical mode decomposition: a novel technique for the study of tremor time series. Med Biol Eng Comput 44: 569-582, 2006

Rosenberg JR, Amjad AM, Breeze P, Brillinger DR, Halliday DM. The Fourier approach to the identification of functional coupling between neuronal spike trains. Prog Biophys Mol Biol 53: 1-31, 1989.

Schmied A, Ivarsson C, Fetz EE. Short-term synchronization of motor units in human extensor digitorum communis muscle: relation to contractile properties and voluntary control. Exp Brain Res 97: 159-172, 1993.

Sears TA, Stagg D. Short-term synchronization of intercostal motoneurone activity. $J$ Physiol 263: 357-381, 1976.

Semmler JG, Steege JW, Kornatz KW, Enoka RM. Motor-unit synchronization is not responsible for larger motor-unit forces in old adults. $J$ Neurophysiol 84: 358-366, 2000.

Sinkjaer T, Hayashi R. Regulation of wrist stiffness by the stretch reflex. $J$ Biomech 22: 1133-1140, 1989. 